\title{
Energy harvesting from transverse galloping
}

\author{
A. Barrero-Gil *, G. Alonso, A. Sanz-Andres \\ ETSIA Aeronauticos, instituto Universitaija 'Ignacio Da Riva', Universidad Politecnica de Madizd, E-28040 Madizd, Spain
}

\section{A R T I C L E I N F O}

\section{Introduction}

The idea of utilizing wind power to extract energy is not new. However, there is a recent interest in the energy extraction from the flow induced oscillation phenomena. An example is an energy-harvesting eel proposed by Allen and Smith [1]. It consist of a piezoelectric membrane placed in the wake of a bluff body. When the mass and elastic properties of the membrane are appropriate, the vortex street formed behind the body induces significant oscillations in the membrane that can be converted into electricity. Another concept, developed by Tang et al. [2], involves the potential use of flutter for a flexible plate in axial flow. In their paper they analyze the key parameters to be controlled in order to achieve a practical design. The capacity of a flow-energy converter based on an oscillating foil is investigated in Zhu et al. [3]. Inspired by the ability of fish to swim efficiently and extract energy from unsteady flows (to the extent that passive thrust generation becomes feasible in the wake of an upstream object [4]), they consider a foil with two degrees of freedom, heave and pitch, where the pitch motion is imposed by an actuator. The power generation by the heaving motion is both theoretically and numerically investigated. On the experimental side, Simpson et al. [5] demonstrated that foils performing a sinusoidal motion in vertical and rotational way, with continuously controlled parameters such as pitch amplitude, Strouhal number and phase angle between vertical and rotational motion, can efficiently extract energy from the flow.

A very interesting device is the VIVACE (vortex induced vibration aquatic clean energy) converter developed by Bernitsas and Raghawan [6]. The VIVACE converter uses the oscillations induced by vortex shedding from a springmounted circular cylinder in a range of flow velocities. The influence of some key parameters, like the mass ratio (i.e. the dimensionless number typifying the ratio of the mean density of the cylinder to the density of the flow), the mechanical damping, the Reynolds number, and the aspect ratio cylinder's (length to diameter ratio) are investigated. 
Here we discuss for the first time the use of galloping as an alternative to extract energy from the flow. Transverse galloping (TG) is well known in the civil-engineering field (see [7]), as it is observed in high-tension electric transmission lines when the ice accretion on the wires modifies their initially almost circular sections promoting oscillations of the wires [8|. Basically, TG consists of a movement-induced vibration appearing in some elastic bluff bodies when the velocity of the incident flow exceeds a certain critical value. Then, the stabilizing effect of structural (mechanical) damping is overcome by the destabilizing effect of the fluid force, and a small transverse displacement of the body creates a fluid force in the direction of the motion that tends to increase the amplitude of vibration. Once the instability threshold is exceeded an oscillatory motion (mainly transverse to the flow) develops with increasing amplitude until the energy dissipated per cycle by mechanical damping balances the energy input per cycle from the flow. If the elastic properties are appropriate this steady amplitude of oscillations can be many times the characteristic transverse dimension of the body.

Den Hartog was the first one in establishing the conditions for the onset of TG using the quasi-steady hypothesis to describe the linearized fluid forces. The assumption considers that the instantaneous fluid force is the same as when the body is stationary at the same relative vector velocity (angle of attack). This is particularly a good approximation at high flow velocities, when the characteristic timescale of the flow is small compared to the characteristic timescale of oscillation. The evolution of the body once galloping is started as well as some nonlinear features of the phenomenon, like its hysteretic behavior, are wel] discussed in Parkinson [9]. Contrary to the vortex induced vibration phenomenon, where significant oscillations develop in a small range of flow velocities and with limited oscillation amplitudes, galloping occurs for an infinite range of flow velocities and without a self-limited response beyond the critical flow velocity (in the sense that as the flow velocity increases the amplitude of oscillation increases too). With the idea of extracting energy from the flow this is a clear advantage, because if the instability appears at moderate flow velocities, the device could be oscillating (and therefore generating energy) from low to high flow velocities. To this end, a good selection of geometrical and mechanical properties is needed as it will be shown appropriately later. In this paper we analyze theoretically the feasibility of using TG to extract energy from a fluid flow. To describe the fluid force the quasi-steady approach is used, and the role in the efficiency factor of some key parameters is investigated. A relevant result of the analysis is that the maximum attainable efficiency (defined as the ratio of the power from the flow to the body and the total power in the flow) depends solely on the cross-section geometry.

Following a description of the mathematical modelling of transverse galloping in the next section (Section 2), we study the efficiency dependence on the cross-section geometry and the mechanical properties (Section 3). Section 4 is devoted to discuss and propose a prototype and, finally, some conclusions are drawn in Section 5.

\section{Energy transfer and conversion factor}

\subsection{Mathematical model of TG}

Let us consider a simplified configuration which consists of a spring-mounted prismatic body prone to gallop under the action of an incoming flow in the transverse direction (see sketch in Fig. 1). It has a mass per unit length $m$, mechanical damping ratio $\zeta$ and natural circular frequency of oscillations $\omega_{N}$. Moreover, the body is sufficiently slender to consider bidimensional flow, and the incident flow is free of turbulence. Then, the equation governing the dynamics of the system is

$$
m\left(\ddot{y}+2 \zeta \omega_{N} \dot{y}+\omega_{N}^{2} y\right)=F_{y}=\frac{1}{2} \rho U^{2} D C_{y}
$$

where $y$ denotes the vertical position, $\rho$ is the fluid density, which will be considered constant throughout the analysis, $U$ is the undisturbed velocity of the incident flow, $D$ is the characteristic dimension of the body normal to the flow, $F_{y}$ is the

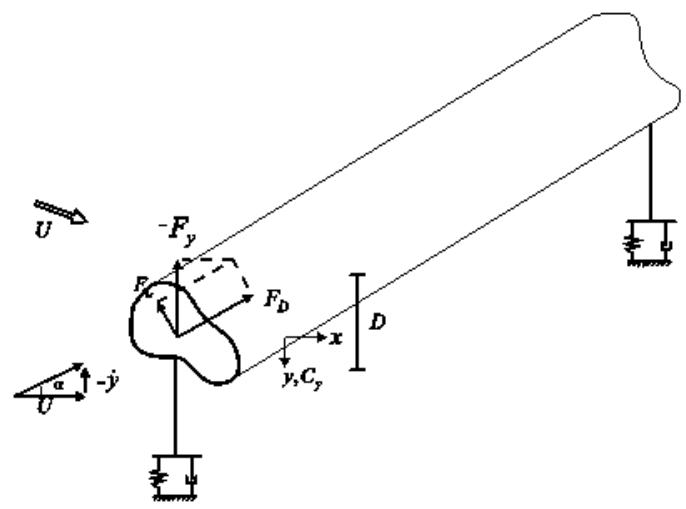

Fig. 1. Fluid forces on the cross-section and the angle of attack induced by the oscillation. 
fluid force per unit length in the normal direction to the incident flow, $C_{y}$ is the instantaneous fluid force coefficient also in the transverse direction to the incident flow, and, finally, the dot symbol stands for differentiation with respect to time $t$.

Usually, the transverse galloping phenomenon is characterized by a timescale of the body oscillation $\left(\sim 2 \pi / \omega_{N}\right)$ much larger than the characteristic timescale of the flow $(\sim D / U)$, so that the fluid force can be evaluated by using the quasisteady hypothesis [10]. Since $\tan (\alpha)=\dot{y} / U$ (see Fig. 1), the fluid force is an empirical function of $\dot{y}$, which can be approximated by a polynomial when the static variation of $C_{y}$ with $\alpha$ is known. Observe that $C_{y}$ can be related to the lift and drag coefficients $C_{L}$ and $C_{D}\left[C_{y}=-\left(C_{L}+C_{D} \tan \alpha\right) / \cos \alpha\right]$. For our purposes a cubic polynomial can be used to approximate the vertical fluid force coefficient,

$$
F_{y}=\frac{1}{2} \rho U^{2} D\left(a_{1} \frac{\dot{y}}{U}+a_{3}\left(\frac{\dot{y}}{U}\right)^{3}\right)
$$

where $\mathfrak{a}_{1}$ and $\mathfrak{a}_{3}$ are empirical coefficients to fit by a polynomial the $C_{y}$ versus $\tan (\alpha)$ dependence measured in static tests (note that if the cross-section is symmetric about a line in the direction of the flow through the center of the section, only odd harmonics, $a_{1}, a_{3}$, etc. in the series are nonzero [11]). The linear coefficient $a_{1}=-\left(\partial C_{1} / \partial \alpha+C_{D}\right)$ is the slope of the vertical fluid force coefficient at zero angle of attack. For galloping it is necessary that $a_{1}>0$ and, therefore, the slope of the lift coefficient must be negative (this point will be discussed appropriately later). $a_{3}$ accounts for the nonlinear dependence of $C_{y}$ with $\alpha$ and it is negative (note that $C_{y}$ cannot increase with $\alpha$ without limit). Both coefficients $\mathfrak{a}_{1}$ and $\mathfrak{a}_{3}$ show a dependence on several factors, namely the cross-section geometry, but also on the aspect ratio of the body $L D$ or the characteristics of the incident flow.

Introducing dimensionless variables $\eta=y / D$ and $\tau=\omega_{N} t$, and taking into account the expression developed for $F_{y}$ in Eqs. (2), (1) becomes

$$
\eta^{\prime \prime}+2 \zeta \eta^{\prime}+\eta=\frac{U^{* 2}}{2 m^{*}}\left(a_{1} \frac{\eta^{\prime}}{U^{*}}+a_{3}\left(\frac{\eta^{\prime}}{U^{*}}\right)^{3}\right)
$$

where the prime represents differentiation with respect to the dimensionless time $\tau$ and $m^{*}=m / \rho D^{2}$ is the dimensionless mass ratio (i.e. the ratio of the mean density of the body to the density of the surrounding fluid), and $U^{*}=U /\left(\omega_{N} D\right)$ is the reduced velocity.

\subsection{Galloping response}

Eq. (3) can be solved either numerically or by asymptotic methods if the nonlinear term is small. In the case that both aerodynamic and damping forces, of order $U^{\prime \prime} / \mathrm{m}^{*}$ and $\zeta$, respectively, are small compared with inertia and stiffness forces (of order unity in the dimensionless equation), solutions to Eq. (3) will tend to a limit cycle of quasi-harmonic oscillations with normalized amplitude $A^{*}=A / D$ ( $A$ is the amplitude of oscillations). This behavior is quite usual for elastic bodies in air, where $m^{*}$ is typically of order $10^{3}$.

Employing the Krylov-Bogoliuvov method to solve approximately Eq. (3) one can find (see Appendix or [12]) the normalized amplitude of oscillations as a function of the cross-section geometry ( $a_{1}$ and $a_{3}$ ), flow velocity and mass and mechanical properties (synthesized in the product $m^{*} \zeta$, the so-called mass-damping parameter),

$$
A^{*}=\left(\frac{4 U^{*}}{3 a_{3}}\left(4 m^{*} \zeta-a_{1} U^{*}\right)\right)^{1 / 2}
$$

It can be seen from Eq. (4) that only for $U^{*}>U_{g}^{*}=4 m^{*} \zeta / a_{1}$ (the critical velocity of galloping) $A^{*}$ is well defined (remember that $a_{1}>0$ and $a_{3}<0$ ). Observe that $U_{\mathrm{g}}^{*}$ can also be deduced from the linearized version of Eq. (3); at $U_{\mathrm{g}}^{*}$ the destabilizing effect of the fluid force equals the stabilizing effect of mechanical damping. We see therefore that galloping is only possible for some bluff bodies, where the flow is stalled (it is distinguished by two shear layers rolling up in vortices forming a broad wake) and the $C_{y}$ slope can be positive for low values of the induced angle of attack $\left(a_{1}>0\right)$. For larger values of the angle of attack the lower shear layer reattaches to the body and the $C_{y}$ slope becomes negative $\left(a_{3}<0\right)$. However in the situation of non-separated flow as the angle of attack grows the $C_{y}$ slope becomes negative and galloping does not occur. One may also check from Eq. (4) jf the quasi-steady hypothesis can be used. Observe that for very low values of $m^{*} \zeta$ galloping appears at low velocities (probably combined with vortex-induced vibrations) and the quasi-steady hypothesis is without physical sense.

\subsection{Conversion factor}

It is possible to introduce a conversion factor (or efficiency) defined by the ratio of the power imparted by the flow to the body per unit length and the total power in the flow per unit length, that is

$$
\eta_{I}=P_{F-B} / P_{F}
$$


where the total power in the flow per unit length is $\rho U^{3} \mathrm{D} / 2$. The power extracted from the flow by the oscillating body, per cycle of oscillation $T$ and per unit length, is given by

$$
P_{F-B}=\frac{1}{T} \int_{0}^{T} F_{y} \dot{y} \mathrm{dt}
$$

Considering sinusoidal oscillations with amplitude $A$ and frequency $\omega_{N}\left(y=A \sin \omega_{N} t\right)$, it follows from Eqs. (2) and (6) that the conversion factor $\eta_{1}$ can be expressed in terms of the normalized amplitude and reduced velocity as

$$
\eta_{1}=\frac{a_{1}}{2}\left(\frac{A^{*}}{U^{*}}\right)^{2}+\frac{3 a_{3}}{8}\left(\frac{A^{*}}{U^{*}}\right)^{4}
$$

Note that the first term of the right is positive and the second term negative ( $a_{1}>0, a_{3}<0$ ). Finally, introducing Eq. (4) in Eq. (7) one obtains

$$
\eta_{j}=2 a_{1}\left(\frac{4 m^{*} \zeta-a_{1} U^{*}}{3 a_{3} U^{*}}\right)+6 a_{3}\left(\frac{4 m^{*} \zeta-a_{1} U^{*}}{3 a_{3} U^{*}}\right)^{2}
$$

which contains the influence of cross-section properties, mass and elastic properties, and flow velocity in the conversion factor.

\section{Role of the cross-section geometry and mechanical properties in the conversion factor}

Fig. 2a and $\mathrm{b}$ shows the normalized amplitude of oscillations and the efficiency factor dependence with the geometrical characteristics of the cross-section and the flow velocity. The mass-damping parameter $m^{*} \zeta$ and the first aerodynamic coefficient $a_{1}$ are fixed, taking $a_{3}$ three different values. Observe that $m^{*} \zeta$ and $a_{1}$ has the same values in all cases and, therefore, the reduced velocity at which galloping starts is the same in all cases (remember that $U_{g}^{*}=4 \mathrm{~m}^{*} \zeta / a_{1}$ ). As it is seen in Fig. 2a, once galloping is started, the normalized amplitude of oscillations increases with the reduced velocity, more dramatically for low values of $a_{3}$. Conversely, Fig. $2 \mathrm{~b}$ shows the conversion factor (or efficiency) evolution with the reduced velocity. Beyond the critical velocity of galloping the conversion factor increases until a maximum value is reached. Then, the conversion factor diminishes slowly asymptotically to zero (as can be deduced from Eq. (7)). It seems that the maximum efficiency is reached at the same reduced velocity in all cases.

Fig. 3a shows the conversion factor as a function of the reduced velocity for given values of $m^{*} \zeta$ and $a_{3}$. Galloping starts at different velocities in all cases due to the variable value of $a_{1}$. Also, one can note the different velocity at which the efficiency is maximum in all cases. Fig. 3b shows the conversion factor dependence on $a_{1}$ and $\mathfrak{a}_{3}$ for fixed values of the mass and elastic properties and flow velocity. Based on this figure, it seems clear that in order to improve the power extraction it is needed to look for a cross-section with high values of $a_{1}$ and low absolute values of $a_{3}$. It should be noted here that the values given in Figs. 2 and 3 are realistic, and they are based in our measurements of the $C_{y}(\alpha)$ curve for isosceles triangles (see Table 1 for details).

The role of the mass and mechanical properties is shown in Fig. 4, where the conversion factor for three different values of the mass-damping parameter can be observed. As expected, the flow velocity at which galloping starts depending

(a)

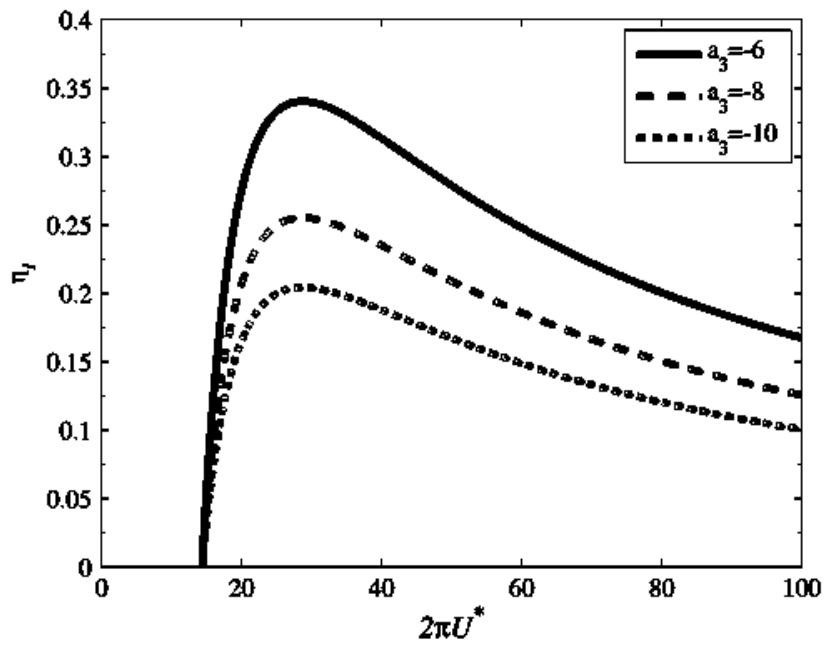

(b)

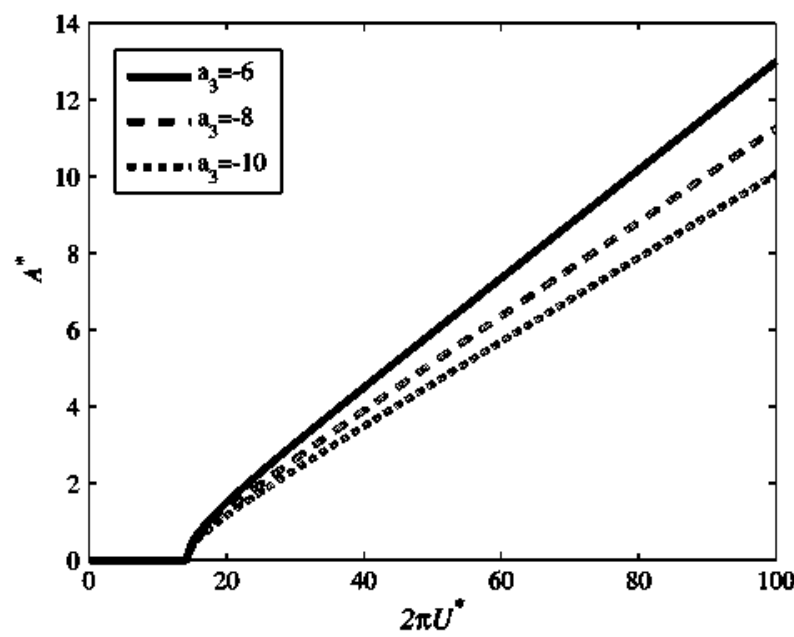

Fig. 2. Influence of cross-section geometry. (a) Influence of $a_{3}$ in the conversion factor ( $a_{1}-3.5$ is fixed). (b) Corresponding amplitude of oscillations. The mass and elastic properties are fixed and the mass-damping parameter takes a value of $m^{*} \zeta=2$. 
(a)

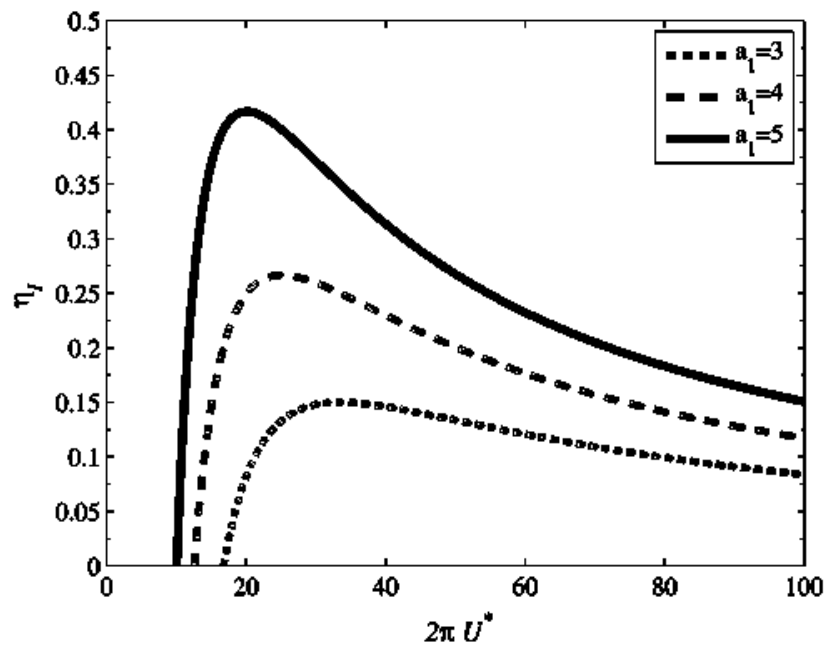

(b)

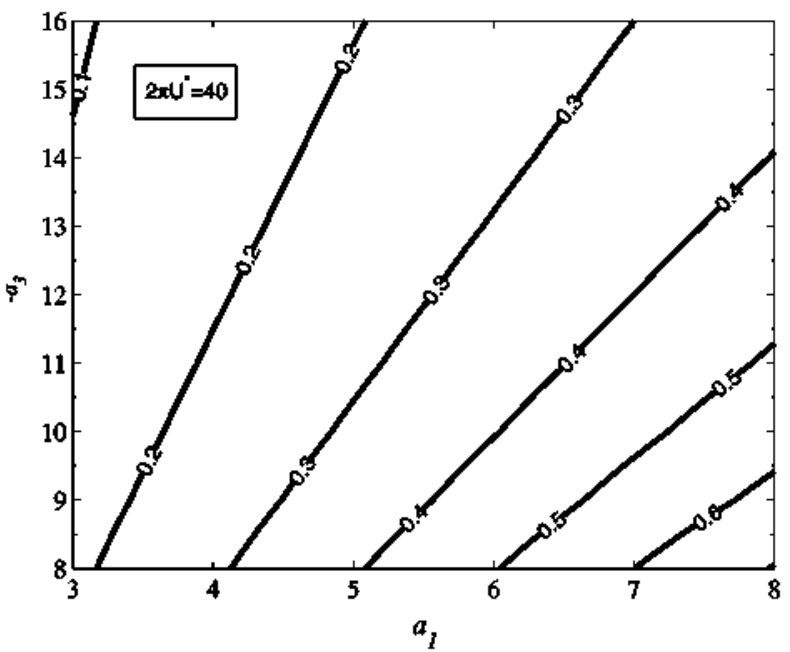

Fig. 3. Influence of cross-section geometry. (a) Influence of $a_{1}$ in the conversion factor ( $a_{3}=-10$ is fixed) for a fixed value of the mass-damping parameter, $m^{*} \zeta=2$. (b) lnfluence of $a_{1}$ and $a_{3}$ in the conversion factor for a fixed value of $m^{*} \zeta=2$, as well as the normalized velocity, being $2 \pi U^{*}=40$.

Table 1

Static aerodynamic characteristics of a square and isosceles cross-sections.

\begin{tabular}{|c|c|c|c|c|}
\hline Cross-section & $a_{1}$ & $a_{3}$ & $\eta_{1 \max }=-a_{1}^{2} / 6 \mathbf{a}_{3}$ & Source and comments \\
\hline Square & 2.3 & -18 & 0.05 & $|13| ; 33000<\operatorname{Re}<66000 ; \mathrm{Tu} \approx 0$ \\
\hline Isosceles triangle $\left(\delta=30^{\circ}\right)$ & 2.9 & -6.2 & 0.25 & {$\left[14\right.$ ] $: \operatorname{Re} \approx 10^{5} ; T u=4 \%$} \\
\hline D-section & 0.79 & -0.19 & 0.54 & [15]: $\operatorname{Re} \approx 10^{5} ; \mathrm{Tu}=11 \%$ \\
\hline Isosceles triangle $\left(\delta=53^{\circ}\right)$ & 1.9 & 6.7 & 0.09 & $|16|: \operatorname{Re} \approx 10^{4}$ \\
\hline
\end{tabular}

Tu indicates the intensity of turbulence and $\delta$ is the angle of the different measure for the isosceles triangle.

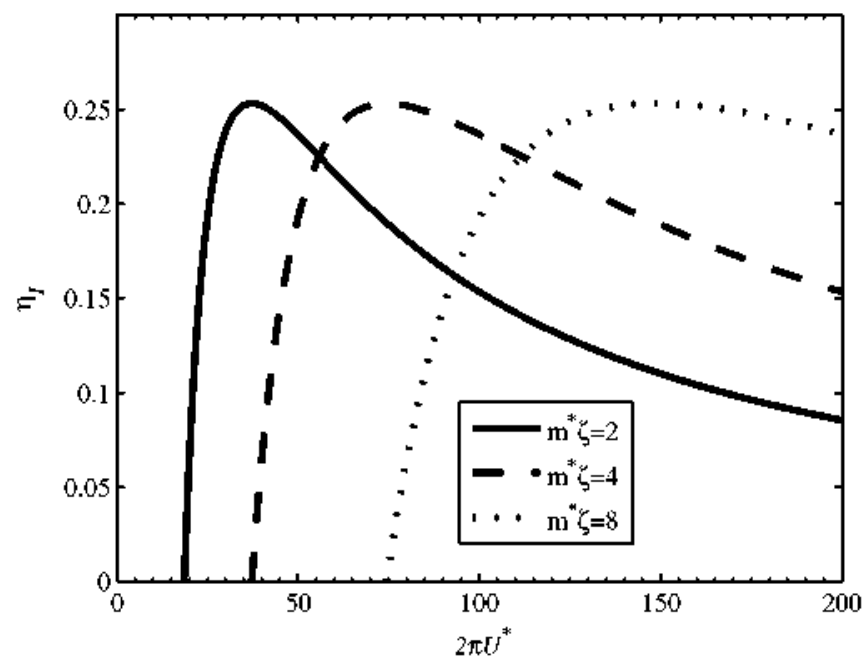

Fig. 4. Influence of the mass and elastic properties. $a_{1}=2.7, a_{3}=-4.8$ ( see Table 1 ).

strongly on the mass-damping parameter (the lower $m^{*} \zeta$ is, the lower the critical velocity of galloping is), but it seems that the maximum efficiency attainable is independent on that parameter. On the other hand, the velocity at which the maximum efficiency is achieved can be deduced by taking the first derivative of Eq. (8) with respect to the reduced velocity and equating to zero. After a little bit of algebra one obtains

$$
\frac{\partial \eta_{1}}{\partial U^{*}}=-\frac{8 m^{*} \zeta\left(8 m^{*} \zeta-a_{1} U^{*}\right)}{3 a_{3} U^{* 3}},
$$




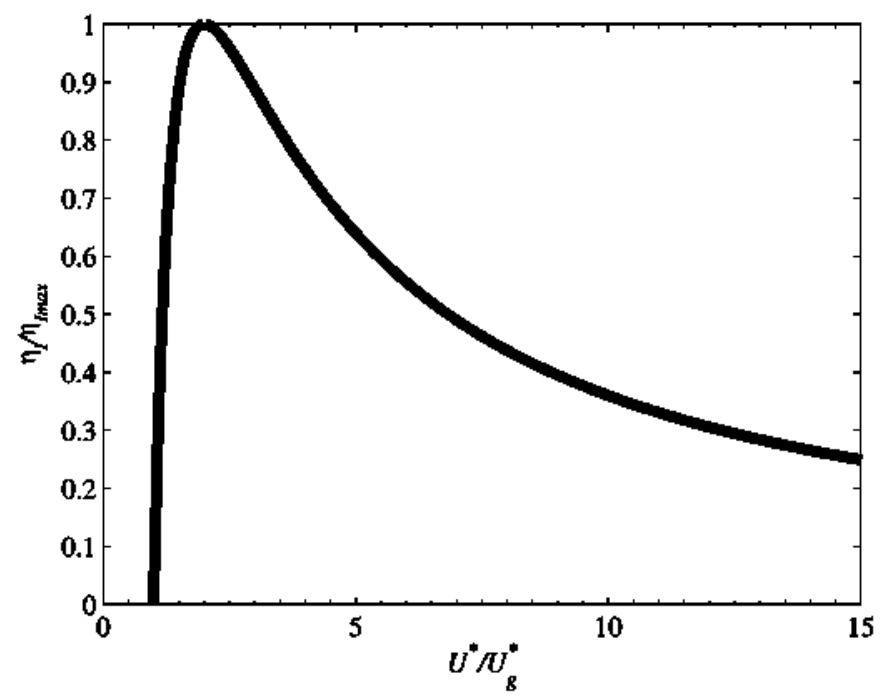

Fig. 5. Universal plot of the efficiency versus the flow velocity.

and therefore,

$$
U^{*}\left(\eta_{\operatorname{lmax}}\right)=\frac{8 m^{*} \zeta}{a_{1}}=2 U_{g}^{*}
$$

where $U_{g}^{*}=4 m^{*} \zeta / a_{1}$ is the reduced velocity at which galloping starts. The reduced velocity at which the efficiency is maximum depends only on the mass-damping parameter and the first fluid coefficient $\mathfrak{a}_{1}$. Substituting $U^{*}\left(\eta_{l_{\max }}\right)$ in $\mathrm{Eq} \mathbf{q}_{+}(8)$ it follows that

$$
\eta_{\max }=-\frac{a_{1}^{2}}{6 a_{3}} .
$$

This is a relevant result (as Fig. 4 anticipates), as the maximum efficiency depends exclusively on the cross-section geometry, being independent of the mass and mechanical properties of the prism. Then, the efficiency dependence shown in Fig. 4 can be redrawn beautifully (see Fig. 5 ) in a universal curve introducing the normalized variables $\tilde{\eta}=\eta_{/} / \eta_{\operatorname{lnax}}$ and $\tilde{U}=U^{*} / U_{g}^{*}$, being $\eta_{I \max }=-\mathfrak{a}_{1}^{2} /\left(6 a_{3}\right)$ and $U_{g}^{*}=4 m^{*} \zeta / a_{1}$. After a little of algebra one gets,

$$
\ddot{\eta}=-4 Z(1-Z)
$$

being $Z=(1-\tilde{U}) / \tilde{U}$.

\subsection{Harnessable energy}

The power that can be converted into electricity is the power extracted from the flow minus the power dissipated in the different transmission stages. To simplify the case one can assume that internal losses includes both the energy extracted by the electric generator (leading to a generator-associated damping, $\zeta_{g}$ ) and the mechanical damping $\zeta$. Therefore, the harnessable energy $P_{\mathrm{HE}}$ per unit length can be computed from Eq. ( 8 ) substituting the mechanical damping, $\zeta$ by the total damping $\zeta_{T}=\zeta+\zeta_{g}$ if the electric system is considered. That is.

$$
P_{\mathrm{HE}}=\rho U^{3} D\left[a_{1}\left(\frac{4 m^{*} \zeta_{T}-a_{1} U^{*}}{3 a_{3} U^{*}}\right)+3 a_{3}\left(\frac{4 m^{*} \zeta_{T}-a_{1} U^{*}}{3 a_{3} U^{*}}\right)^{2}\right] \frac{\zeta_{g}}{\zeta_{T}} .
$$

It should be noted that if the total damping is very high the asymptotic analysis presented here can be a poor approximation and a numerical solution to Eq. (3) may be needed.

\section{Discussion about the practical implementation. GECO converter}

From the practical side the question is how much power can be extracted given a specific design. The theory developed in Section 2 gives the designer a means of examining how the power developed by a prototype is governed by the various design parameters. The performance of the prototype can be characterized by the manner in which the power vary with the wind speed or in terms of the power extracted by unit of area. A prototype for the galloping energy converter (GECO) could consists of a spring mounted prism with constant cross-section and length $L$. Moreover, the device must incorporate a system to convert the oscillating motion of the prism into electricity. Here we will not discuss in detail the method to do 
that, but roughly speaking some alternatives are possible, like using a rod-crank mechanism to get a circular motion and then connecting to a generator, or using a piezoelectric material where the deformation is directly transduced into a voltage.

\subsection{Power per wuit length}

Expressions (10) and (11) constitute a guide for the prototype. It is clear that one must look for a cross-section with the highest value of $-a_{1}^{2} / a_{3}$ to develop high efficiency, but what about the mass and mechanical properties? On one hand, a low value of the mass-damping parameter involves that the flow velocity at which galloping starts is low (note also the role of $a_{1}$ in the critical velocity of galloping, $\left.U_{g}^{*}=4 m^{*} \zeta / a_{1}\right)$, but on the other hand the range of flow velocities at which the efficiency is high is reduced (see Figs. 4 and 5). Necessarily, there is a compromise between getting a low critical velocity of galloping and getting high efficiency in a large range of flow velocities. The engineering solution must be based on the specific wind characteristics to be installed, as the quantity of energy that can be captured depend upon the power versus wind speed characteristics and the wind speed distribution of the site where GECO is planned.

Fig. 6 shows the fluid force coefficient measured in wind tunnel for a square, isosceles triangle, and D-type crosssections (for $\alpha=0$ the flow is aligned with its longitudinal axis), and Table 1 presents the fluid force coefficients for a twoterm polynomial approximation of $C_{y}$ versus $\tan (\alpha)$. To illustrate the power captured as a function of the wind speed, let us consider the case of an isosceles triangle $\left(\delta=30^{\circ}\right)$ cross-section with transversal dimension $D=0.15 \mathrm{~m}$ and supported elastically with a natural frequency of oscillations of $1 \mathrm{~Hz}$. As it can be seen in Fig 7 , for $\mathrm{m}^{*} \zeta=2$, there is no flow energy extraction for $U<3 \mathrm{~m} / \mathrm{s}$, but for $U=10 \mathrm{~m} / \mathrm{s}$ the device would be extracting $18.4 \mathrm{~W} / \mathrm{m}$, and $46.5 \mathrm{~W} / \mathrm{m}$ for $U=15 \mathrm{~m} / \mathrm{s}$. For a higher mass ratio, $m^{*} \zeta=5$ there is no transfer of energy until a flow velocity of $U=7 \mathrm{~m} / \mathrm{s}$, but for $U=10 \mathrm{~m} / \mathrm{s}$ the device would be extracting 19.2 and $76.5 \mathrm{~W} / \mathrm{m}$ for $U=15 \mathrm{~m} / \mathrm{s}$. The $P$ versus $U$ curve has a higher curvature for a larger value of the massdamping parameter and, therefore, it seems reasonable to think that in sites with frequent strong winds it would be preferable to design the device with a high value of the mass-damping parameter.

Based on Table 1, one might think that the best choice for TG energy harvesting could be a D-section, as the maximum efficiency attainable is as high as 0.54 . But the question is not so simple, due to its low value of $a_{1}$ making necessary high velocities for the incident flow. Of course, once the critical velocity is exceed the energy extraction is very high, as it can be seen in Fig. 8 . In places with strong winds probably a D-section would be the best choice.

\subsection{Power extracted per unit of area}

It is interesting to analyze the theoretical behavior of the prototype in terms of power extraction per unit of area. In this sense it is important to define what is considered as area. Here, we consider the area (normal to the wind) covered by the prism in its oscillation, as this is the minimal working area for the oscillating prism; that is $S=(2 A+D) L$ being $A$ the amplitude of oscillations. Regarding this aspect, it is important the size of the cross-section and the frequency of oscillation. Fig. 9 gives a sample set of the results obtained with the aid of Eqs. (4) and (8) and shows the power per unit of area at $U=10 \mathrm{~m} / \mathrm{s}$ as a function of the mass-damping parameter, size of the cross-section, $D$, and natural frequency of oscillations $f_{N}$. The power density $P_{S}$ is defined as $P_{S}=P /(2 A+D)$, $P$ being the extracted power per unit length.

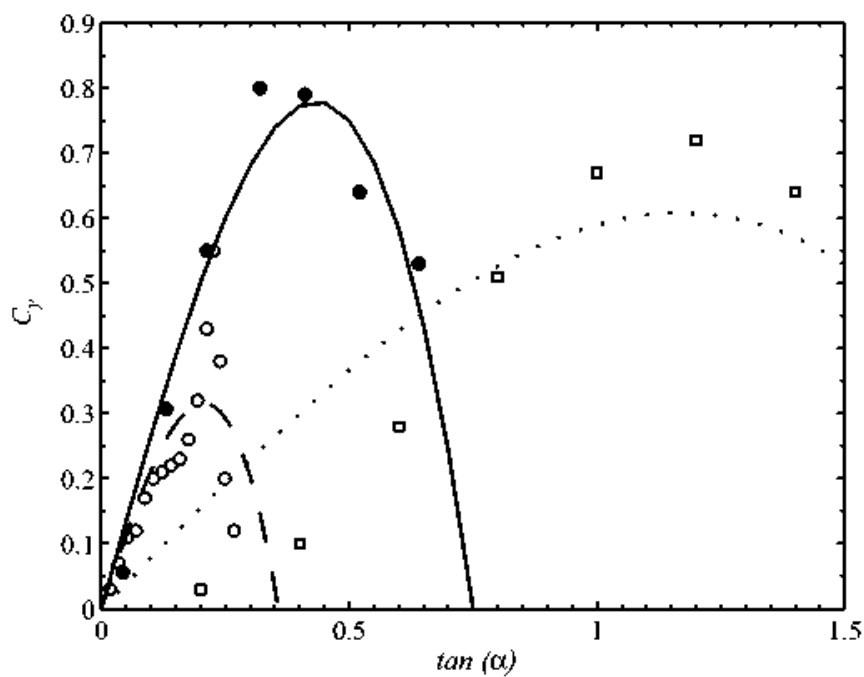

Fig. 6. Steady transverse force coefficient for a square (white circles), isosceles triangle (black circles) and D-section (open squares) cross-sections. lines correspond to the fitting curves. 
(a)

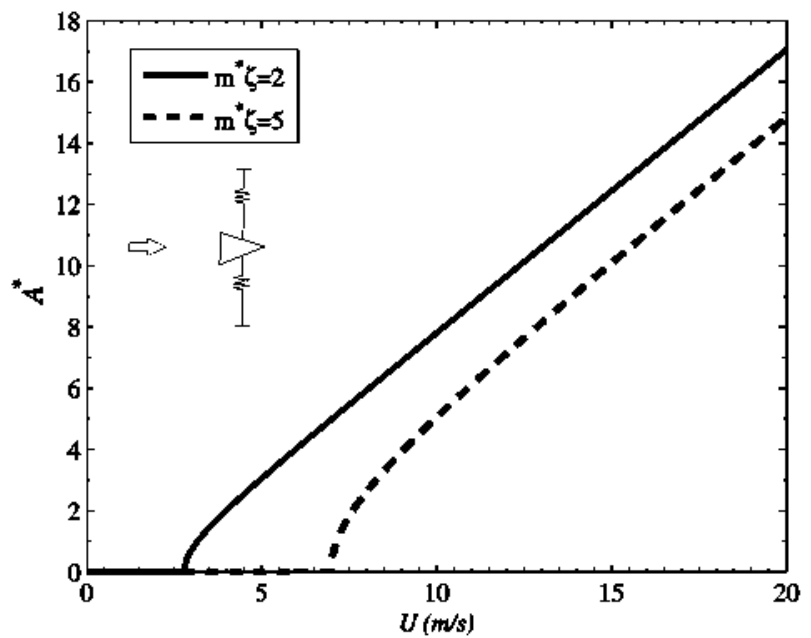

(b)

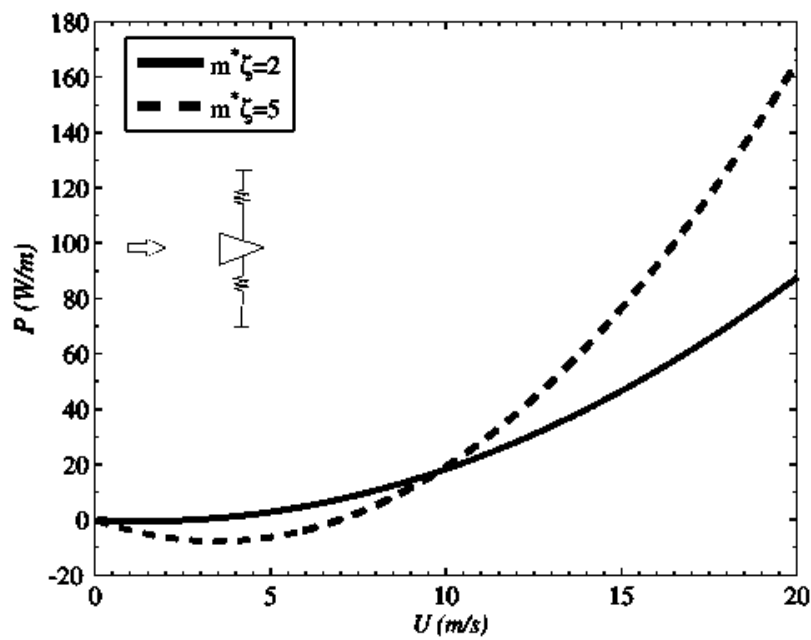

Fig. 7. Theoretical behavior of the prototype (isosceles triangular section) operating under steady conditions for different flow velocities and massdamping parameter. (a) Normalized amplitude of oscillations. (b) Power extracted per unit length.

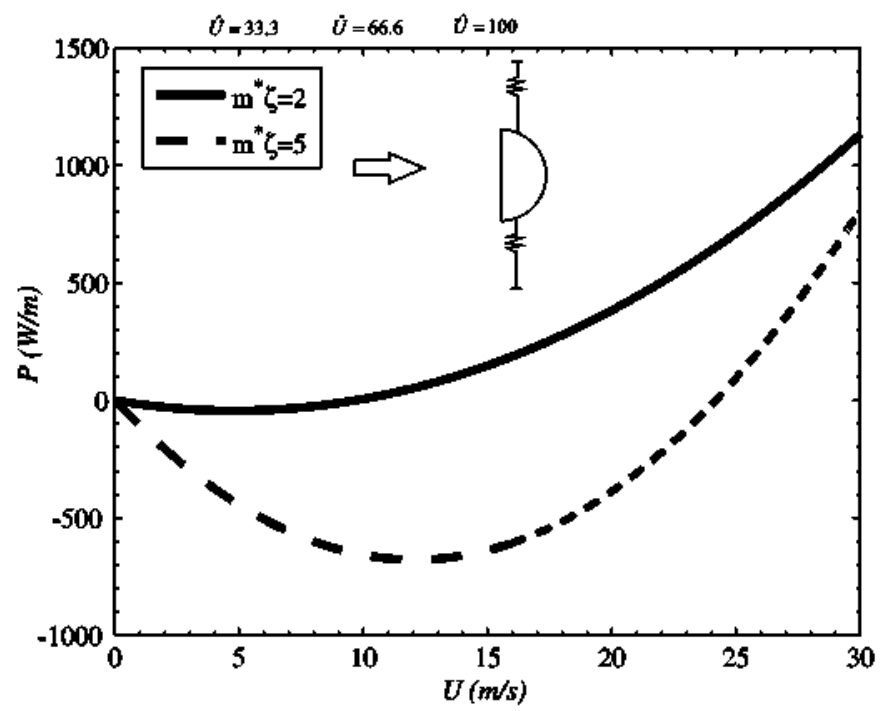

Fig. 8. Theoretical power per unit length extracted by a prototype with a D-type cross-section. It is also shown representative values of the reduced velocity $\left(\hat{U}=2 \pi U^{*}\right)$ to see when the quasi-steady hypothesis holds.

It is seen in Fig. 9 that low values of the mass-damping parameter are needed in order to increase the extracted power per unit of area. Regarding the frequency of oscillation and size of the cross-section it seems that the diameter and the frequency of oscillation has opposite effects. For a high value of the frequency of oscillation, the optimum diameter is lower that for the case of low values of frequency. At this velocity, $U=10 \mathrm{~m} / \mathrm{s}$, observe that theoretical values of the order of 40 W/ $/ \mathrm{m}^{2}$ can be achieved for a damping-parameter value of $1\left(\mathrm{D}=0.08 \mathrm{~m}\right.$ and $f_{N}=10 \mathrm{~Hz}$ ). This is a poor result compared with an horizontal axis wind turbine with a diameter of $17 \mathrm{~m}$ working at a constant speed of $44 \mathrm{rpm}$ ( see [17, p. 183|) which can achieve a value of order $200 \mathrm{~W} / \mathrm{m}^{2}$ at a wind velocity of $10 \mathrm{~m} / \mathrm{s}$. In terms of efficiency, observe that $\mathrm{jf}$ the efficiency is defined as $\eta_{S}=2 P /\left[\rho U^{3}(2 A+D)\right]=2 P_{S} /\left(\rho U^{3}\right)$ it takes a value of 0.067 . This is a low value compared to the Betz coefficient (at around 59 percent) and when compared to other methods to generate energy, like the one base on a coupled heaving/ pitching foil. For example, Simpson et al. reports efficiencies (defined in this last way) as high as 0.43 . However, we believe that GECO could constitute an alternative in situations where wind turbines do not operates efficiently, like for low and high wind speed, or in places where wind turbines (and other energy extraction methods) cannot be installed.

Finally, observe that plots shown in Fig. 9 are also a useful tool for the design of a GECO. For example if one needs 25 $W / \mathrm{m}^{2}$ at a given velocity of wind of $10 \mathrm{~m} / \mathrm{s}$ one has several possibilities like the ones marked by points P1 and P2 in this figure. If one chose the first option (P1) and assume a damping value of 0.1 then the mass of the prism must be $\mathrm{m}=4.4 \mathrm{~kg} / \mathrm{m}$ 
(a)

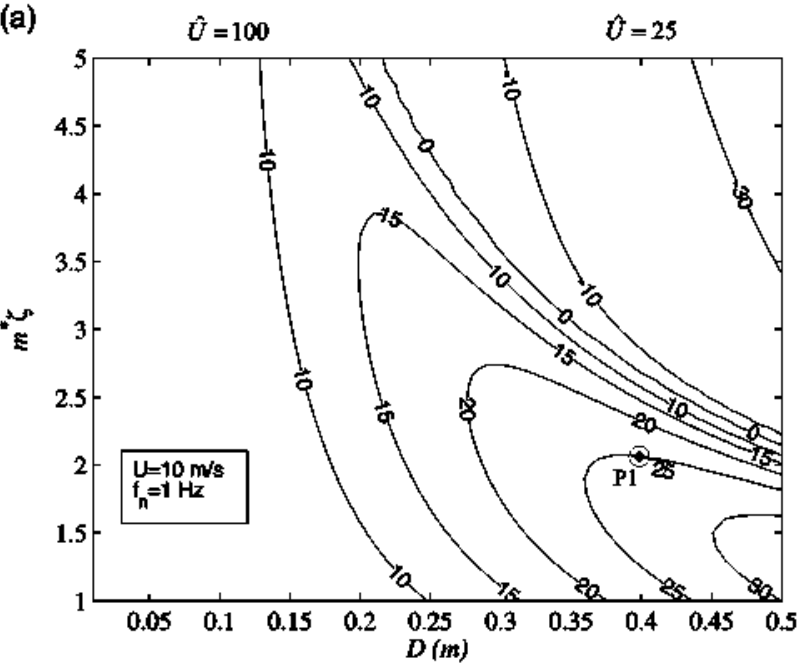

(c)

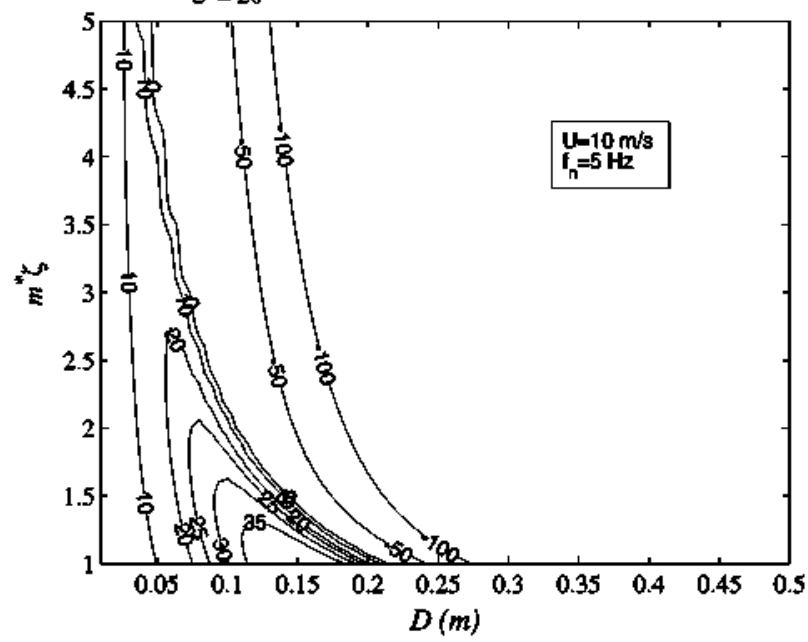

(b)

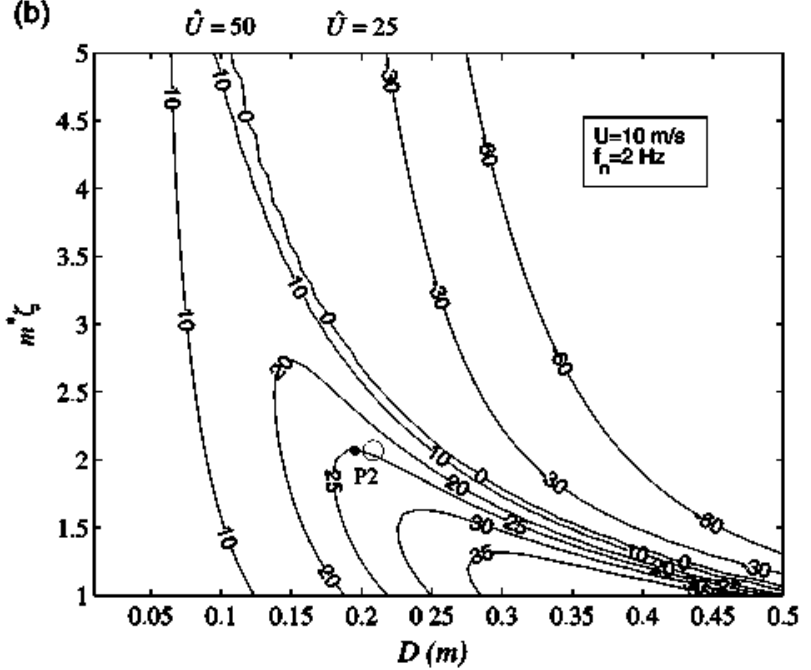

(d)

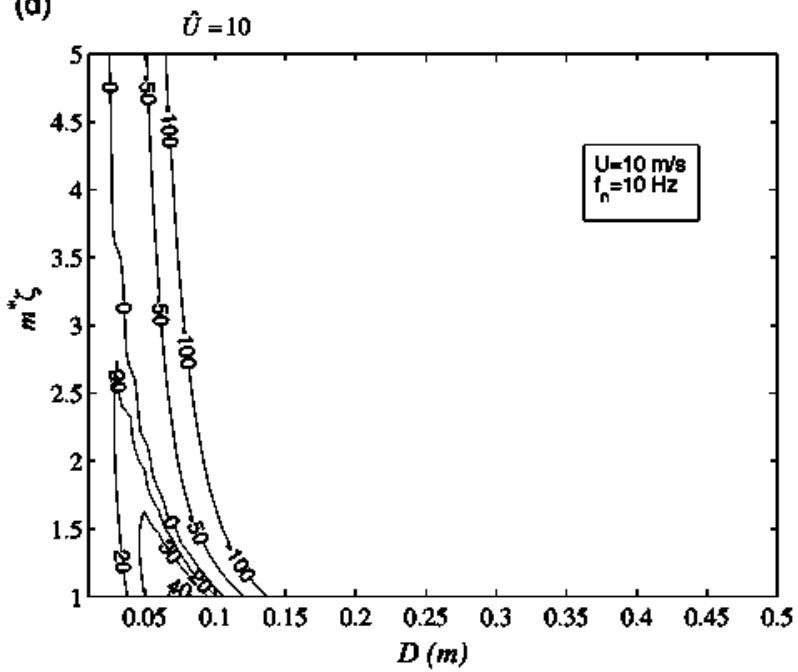

Fig. 9. Theoretical power per unit area extracted at $U=10 \mathrm{~m} / \mathrm{s}$ by a prototype with an isosceles triangle cross-section $\left(\delta=30^{\circ}\right)$ with different size $D$, massdamping parameter, and natural frequency of oscillations. Observe that negative values indicates regions in the space parameter at which galloping does not occur. It is also shown representative values of the reduced velocity $\left(\hat{U}=2 \pi U^{*}\right)$ to see when the quasi-steady hypothesis holds.

$\left(\mathrm{m}^{*} \zeta=2.2\right.$ ), and its dimension $\mathrm{D}=0.4 \mathrm{~m}$. The prism has to be spring-mounted with a natural frequency of $1 \mathrm{~Hz}$ and at a wind velocity of $10 \mathrm{~m} / \mathrm{s}$ the reduced velocity will be $2 \pi U^{*}=25$. For $\mathrm{P} 2: \zeta=0.1, m=1.05 \mathrm{~kg} / \mathrm{m}, D=0.2 \mathrm{~m}, f_{N}=2 \mathrm{~Hz}, 2 \pi U^{*}=25$.

\subsection{Scalability}

For medium or large scale, a practical setup for extracting energy could consist of an array of spring-mounted prisms of appropriate cross-section separated by a given distance (obviously this would be an important parameter of the design that will need to be studied), but as close as possible to minimize the size of the device, in a similar manner as the VIVACE proposal. Due to its apparent simplicity we think that it would be easy to get a robust design, with low cost and long operational life. Also, we believe that TG can be used as well at small scales, and very small elastic prisms could be used to generate electricity. At small scale, the Reynolds number Re (a measure of the relative importance of the inertial fluid forces and viscous forces) emerges as a new parameter in the problem and the coefficients $a_{1}$ and $a_{3}$ will be dependent on that parameter. Therefore, it is expected that the scaling law with the size of the elastic body for the harnessable energy will not be directly extrapolable from Eq. (13). Actually, this scenario is being investigated both theoretically and experimentally for $\operatorname{Re} \sim 10^{2}[18 \mid$. Taking advantage of other flow induced vibrations some work has been developed at this small scale [19]. 


\section{Conclusion}

This paper considers for the first time the TG phenomenon to extract energy from the flow. Based on a theoretical model of $\mathrm{TG}$, the relation between the mass and mechanical properties, cross-section geometry, flow velocity and energy efficiency (or conversion factor) has been established. Some relevant relationships, like the sole dependence of the maximum attainable efficiency on the cross-section geometry, or the flow velocity at which maximum efficiency takes place have been found. The analysis also serves as a starting point to design a prototype working efficiently on a large range of environmental conditions (flow velocity), and a proposed prototype has been discussed. For a good operation it is positive to have: (i) a high value of $a_{1}$, (ii) a low absolute value of $a_{3}$, (iii) low value of the mass-damping parameter, $m^{*} \zeta$, and (iv) the natural frequency of oscillations must be carefully matched in accordance with the size of the cross-section (D) in order to have a good power-area ratio.

With respect to other energy conversion systems, like the ones based on vortex-induced vibrations, where the conversion factor shows a strong dependence with the incoming flow velocity, in this case a device operating in a large range of flow velocities is possible. Finally, it should be noted that the analysis here presented only considers the case $m^{*} \gg 1$ (elastic body in air), but the situation in which $m^{*} \sim 1$ (elastic body in water) is also of interest. Obviously, the latter case demands a numerical approach. Also, other aspects, like the influence of turbulence in the incoming flow must be incorporated for a more realistic approximation.

\section{Appendix A}

The Krylov-Bogoliubov method [20] is particularly useful to obtain approximate solutions for weakly nonlinear oscillators. Let us make a brief summary. Consider the nonlinear harmonic oscillator equation written in dimensionless form

$$
\ddot{x}+x=\varepsilon f(x, \dot{x}), \quad \varepsilon \ll 1
$$

whose solution for the case $\varepsilon=0$ is $X(\tau)=X \cos (\tau+\phi)$ (note that in this case overdots means differentiation respect to the dimensionless time $\tau$ ). For small values of $\varepsilon$, solutions to Eq. (A.1) can be written as $X(\tau)=X(\tau) \cos [\tau+\phi(\tau)]$, where $X(\tau)$ and $\phi(\tau)$ are functions slowly varying with $\tau$ (the effect of the forcing term is small, giving raise to a slow change of the parameters in the harmonic solution). As the non-linearity is small, two different timescales exist in the problem, corresponding to the oscillation and the growth of the amplitude of oscillation (their derivatives with respect of $\tau$ being of order of $\varepsilon$ ). Then, if $x(\tau)=X(\tau) \cos [\tau+\phi(\tau)]$, its derivative with respect to $\tau$ taking into account that $\dot{X} \sim \dot{\phi} \ll 1$, is

$$
\dot{x}(\tau)=-X \sin [\tau+\phi(\tau)],
$$

with the additional condition

$$
\dot{X} \cos [\tau+\phi(\tau)]-X \dot{\phi} \sin [\tau+\phi(\tau)]=0 .
$$

Introducing the derivative of (A.2) with regard to $\tau$ into Eq. (A.1), one finds at the lowest order

$$
\dot{X} \sin [\tau+\phi(\tau)]-X \dot{\phi} \cos [\tau+\phi(\tau)]=\varepsilon f[X \cos (\tau+\phi),-X \sin (\tau+\phi)],
$$

which together with condition (A.3) yields

$$
\begin{aligned}
& \dot{X}=-\frac{\varepsilon}{2 \pi} \int_{0}^{2 \pi} f[X \cos (\tau+\phi),-X \sin (\tau+\phi)] \sin (\tau+\phi) \mathrm{d} \tau, \\
& \dot{\phi}=-\frac{\varepsilon}{2 \pi X} \int_{0}^{2 \pi} f[X \cos (\tau+\phi),-X \sin (\tau+\phi)] \cos (\tau+\phi) \mathrm{d} \tau .
\end{aligned}
$$

Applying this method to Eq. (3) of the paper, we look for solutions in the form,

$$
\eta=A^{*}(\tau) \cos [\tau+\phi(\tau)], \quad \eta^{\prime}=-A^{*}(\tau) \sin [\tau+\phi(\tau)]+O\left(A^{*^{\prime}}\right),
$$

where $A^{*}$ and $\phi$ vary slowly with $\tau$. The application of the Krylov-Bogoljubov method leads to

$$
A^{*^{\prime}}=-\frac{1}{2 \pi} \int_{0}^{2 \pi}\left(-2 \zeta \eta^{\prime}+\frac{U^{* 2}}{2 m^{*}} \sum_{j=1 ; 3} a_{j}\left(\frac{\eta^{\prime}}{U^{*}}\right)^{j}\right) \sin (\tau+\phi) \mathrm{d} \tau
$$

The evaluation of (A.5) is straightforward because $\eta^{\prime}=-A^{*} \sin (\tau+\phi)$ during a cycle of oscillation $(0 \leq \tau \leq 2 \pi)\left(A^{*}\right.$ and $\phi$ are constants). Taking into account that

$$
\begin{gathered}
\frac{1}{2 \pi} \int_{0}^{2 \pi}(\sin x)^{2} \mathrm{~d} x=1 / 2, \\
\frac{1}{2 \pi} \int_{0}^{2 \pi}(\sin x)^{4} \mathrm{~d} x=3 / 8
\end{gathered}
$$


one gets,

$$
A^{*}=\left(-\zeta_{+} \frac{a_{1} U^{*}}{4 m^{*}}\right) A^{*}+\frac{3 a_{3} A^{* 3}}{16 m^{*} U^{*}} .
$$

Finally, the steady amplitude of oscillation is given by the real and positive roots of $A^{*}=0$.

\section{References}

[1] J.J. Allen, A.J. Smits, Energy harvesting eel, Joumal of Fiuids and Structures 15 (3-4) (2001) 629-640.

[2] L. Tang, P. Padoussis, J. Jang, Cantilevered flexible plates in axial flow: energy transfer and the concept of flutter-mill, Joumal of Sound and Vibration $326(1-2)(2009) 263-276$

[3] Q. Zhu, M. Haase, C.H. Wu, Modelling the capacity of a novel flow-energy harvester. Applied Mathematical Modelling 33 (2009) $2207-2217$.

[4] J.C. Ljao, D. Beal, J. Lauder, M.S. Triantafyllou, Fish exploiting vortices decrease muscle activity, Science 302 (5650) (2003) $1566-1569$.

[5] B.J. Simpson, F.5. Hover. M.5. Triantafyllou, Experiments in direct energy extraction through flapping foils, Proceedings of the Eighteenth International Offsiore and Polar Engineering Conference, Vancouver, July 6-11, 2008.

[6] M. Bernitsas, K. Raghawan, VIVACE (vortex induced vibration for aquatic clean energy): a new concept in generation of clean and renewable energy from fluid flow. Journal of Offshore Mechanics and Artic Engineering. ASME Transactions, 2008.

[7] R.H. Scanlan, E. Simiu, Wind Effects on Structures. Fundamentals and applications to design, Wiley, New York, 1996.

[8] J.P. Den Hartog. Mechanical Vibrations, McGraw-Hill, New York, 1056.

[9] G.V. Parkinson, Phenomena and modelling of flow-induced vibrations of bluff bodies, Progress in Aerospace Sciences 26 (2) (1989) $169-224$

[10] E. Naudascher, D. Rockwell, Flow-induced vibrations, An Engineeting Guide, Dover Publications, New York, 1994.

111 R.D. Blevins, Fiow-Induced Vibration. Krieger Publishing Company. Florida, 1990.

[12] A. Barrero-Gil, A. Sanz-Andres, G. Alonso, Hysteresis in transverse galloping: the role of the inflection points, joumal of Fluids and Structures 25 (6) (2009) $1007-1020$

[13] G.V. Parkinson, J.D. Smith, The square prismas an aeroelastic nonlinear oscillator, Orarterly Joumal of Mecianics and Applied Mathematics 17 (1964) $225-239$.

|14| G. Alonso. J. Meseguer. I. Prez-Grande. Galloping stability of triangular cross-sectional bodies: a systematic approach, Journal of Wind Engineering and industizal Aerodymamics 95 (9-11)(2007) 928-940.

15| M. Novak. H. Tanaka. Effect of turbulence on galloping instability, ASCE journol of the Engineering Mechanics Division 100 (1974) $27-47$.

[16] S.C. Luo, Y.T. Chew, T.S. Lee, M.G. Yazdani, Stability to translational galloping vibration of cylinders at different mean angles of attack, joumal of Sound and Vibration 215 (5), (1998) 1183-1194.

|17| T. Burton, D. Sharpe. N. Jenkins, E. Bossanyi, Wind Energy Handbook. Wiley. New York, 2001.

[18] A. Barrero-Gil, A. Sanz-Andres, M. Roura, Transverse galloping at low Reynolds numbers, journal of Fiuids and Structures 25 (7) (2009) $1236-1242$.

|19| G.W. Taylor, J. Burns, S. Kammann. W. Powers, T. Welsh. The energy harvesting eel; a small subsurface ocean/river power generator, IEEE Journai of Oceanic Engineering 26 (4) (2001) 539-547.

[20] ].A. Murdock, Perturbations, Theory and Methods, Wiley, New York, 1991. 